\title{
New predictor of acute necrotizing pancreatitis: Red cell distribution width
}

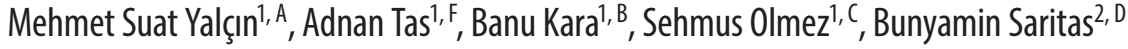 \\ 1 Department of Gastroenterology, Adana Numune Research and Educational Hospital, Turkey \\ ${ }^{2}$ Department of Gastroenterology, Mersin University Medical Faculty, Adana, Turkey \\ A - research concept and design; $B$ - collection and/or assembly of data; $C$ - data analysis and interpretation; \\ $D$ - writing the article; $E$ - critical revision of the article; $F$ - final approval of the article
}

\section{Address for correspondence \\ Adnan Tas \\ E-mail:dradnantas@gmail.com}

\section{Funding sources}

None declared

\section{Conflict of interest}

None declared

Received on June 25, 2016

Reviewed on November 2, 2016

Accepted on December 9, 2016

DOI

$10.17219 /$ acem $/ 67590$

\section{Copyright}

Copyright by Author(s)

This is an article distributed under the terms of the

Creative Commons Attribution Non-Commercial License

(http://creativecommons.org/licenses/by-nc-nd/4.0/)

\begin{abstract}
Background. Acute pancreatitis (AP) is inflammation of the pancreas of various severity ranging from mild abdominal pain to mortality. AP may be classified as acute interstitial edematous pancreatitis (AEP) or acute necrotizing pancreatitis (ANP), according to the revised Atlanta criteria. Most of the patients with AP are AEP (75-85\% of patients), while $15-25 \%$ of patients have ANP. The mortality rate is 3\% in AEP and 15\% in ANP. Thus, it is important to predict the severity of AP to decrease the morbidity and mortality.

Objectives. The aim of the study was to evaluate the relationship between red cell distribution width (RDW) and the severity of AP on admission to hospital.

Material and methods. Patients admitted to Adana Numune Research and Educational Hospital with a diagnosis of AP through the time frame of January 2014-May 2016 were included in our study. Diagnosis of AP was made according to the revised Atlanta classification. Patients' age, sex, etiology of AP, and RDW values were recorded on admission to the hospital.

Results. A total of 180 patients were included in the study. Eighty patients (44\%) were male and 100 patients were female. Mean age was $56.25 \pm 18.3$ years ( $52.66 \pm 14.4$ in males; $59.84 \pm 20.2$ in females). There was no statistically significant difference between patients' age. The most frequently observed etiologic factor was gallstone disease followed by alcohol intake and the use of pharmaceuticals. Drug-related AP was associated with azathioprine, furosemide, and thiazide diuretics. One hundred forty-four (80\%) patients had AEP and 36 (20\%) patients had ANP. RDW values showed a statistically significant difference between patients with AEP and ANP ( $p=0.011$ ). The cut-off value of RDW was 16.4 and the area under curve (AUC) value was 0.591 ( $p=0.0227$ ) with a sensitivity of $29.2 \%$ and specificity of $89.83 \%$.
\end{abstract}

Conclusions. Red cell distribution width could be used to evaluate the prognosis of acute pancreatitis.

Key words: acute pancreatitis, acute necrotizing pancreatitis, red cell distribution width 


\section{Introduction}

Acute pancreatitis (AP) is a disease with inflammation of the pancreas of various severity ranging from mild abdominal pain to mortality. ${ }^{1} \mathrm{AP}$ is characterized by 3 phases. In the $1^{\text {st }}$ phase, enzyme activation and cellular damage cause early symptoms. In the $2^{\text {nd }}$ phase, systemic inflammatory response and intrapancreatic inflammatory reaction occur by the release of proinflammatory and antiinflammatory mediators. In the $3^{\text {rd }}$ phase, complications of AP occur. ${ }^{2} \mathrm{AP}$ is classified as acute interstitial edematous pancreatitis (AEP) or acute necrotizing pancreatitis (ANP) according to the revised Atlanta classification. ${ }^{3}$ AEP constitutes $75-85 \%$ of patients with AP, while ANP consists of $15-25 \%$ of patients with AP. The mortality rate is $3 \%$ in AEP and 15\% in ANP. ${ }^{4}$ Since mortality is 5 times higher in ANP than in AEP, it is important to discriminate ANP from AEP to predict morbidity and mortality. Several scoring systems have been developed to detect the severity of AP. These scoring systems include the Ranson criteria, Acute Physiology and Chronic Health Evaluation II (APACHE II), systemic inflammatory response syndrome criteria, bedside index of severity in acute pancreatitis, harmless acute pancreatitis score, and Balthazar score. ${ }^{5}$ Recent studies have shown newer markers to detect AP severity. These include pancreatic protease activation peptides, interleukin 6 and interleukin 8, polymorphonuclear elastase, procalcitonin, mean platelet volume (MPV), and proteinuria. ${ }^{6,7}$ Red cell distribution width (RDW) is calculated by dividing the standard deviation of red blood cell volume by mean corpuscular volume (MCV) and multiplying it by 100 to express the results as percentages. RDW reflects the variability of the size of the circulating erythrocytes. ${ }^{8}$ Several studies have shown that RDW is significantly associated with inflammatory markers such as C-reactive protein and fibrinogen. ${ }^{9,10}$ In this study, we aimed to evaluate the RDW levels in patients with AEP and ANP.

\section{Material and methods}

The study included 180 patients who were admitted to the gastroenterology department of Adana Numune Research and Educational Hospital (Turkey) with a diagnosis of AP through the time frame of January 2014-May 2016. AP was diagnosed using the revised Atlanta classification. ${ }^{3}$ Patients' age, sex, etiology of AP, and RDW were recorded.

White cell count (WCC), red blood cell (RBC) count, platelet (PLT) count, RDW, hemoglobin (HGB) level, MCV, and mean platelet volume (MPV) were determined using the XE-2100 automated hematology analyser (Sysmex, Kobe, Japan) with Sysmex reagents (Sysmex). The normal reference range for RDW in the laboratory of our hospital is 11.6-15\%.

Exclusion criteria comprised history of chronic pancreatitis or pancreas carcinoma, heart failure, hyperlipidemia, peripheral vascular disease, hematologic disorders, accompanying acute or chronic inflammatory diseases, any other accompanying carcinomas and chronic liver diseases.

\section{Statistical analysis}

Descriptive statistics were used to define the continuous variables and are expressed as mean \pm standard deviation. Student's t-test was used for independent and normally distributed variables. Mann-Whitney U test was used for independent and not normally distributed variables. Receiver operating characteristic (ROC) curve analysis was performed for the variables found to be significant in univariate analysis to determine the cut-off point. Statistical significance level was determined as 0.05 . The analysis was made using MedCalc Statistical Software v. 12.7.7 (MedCalc Software BVBA, Ostend, Belgium).

\section{Results}

A total of 180 patients were included in the study. Eighty patients (44\%) were male and 100 patients $(56 \%)$ were female. Mean age was $56.25 \pm 18.3$ years $(52.66 \pm 14.4$ years for males; and $59.84 \pm 20.2$ years for females). There was no statistically significant difference between the age of male and female patients. Patients were grouped into AEP and ANP. Patients' age was $53.75 \pm 13.3$ years and $55.65 \pm 15$. 6 years in AEP and ANP groups, respectively. There was no statistically significant difference between patients' age.

Patient characteristics are shown in Table 1. The drug-induced AP among the patients was caused by azathioprine, furosemide, and thiazide type diuretics. Imaging studies (ultrasonography, computed tomography and magnetic resonance cholangiopancreatography) and laboratory values revealed no other etiologic factors in the idiopathic pancreatitis group.

One hundred forty-four patients (80\%) were included in the AEP group, and 36 patients (20\%) were included in the ANP group. There was a statistically significant difference in the RDW values between the groups $(\mathrm{p}=0.011)$. There

\begin{tabular}{|c|c|c|c|}
\hline Variable & $\begin{array}{c}\text { AEP } \\
n=144(80 \%)\end{array}$ & $\begin{array}{c}\text { ANP } \\
\mathrm{n}=36(20 \%)\end{array}$ & $\mathrm{p}$-value \\
\hline Age (years) & $53.75 \pm 13.3$ & $55.65 \pm 15.6$ & ns \\
\hline Sex (male/female) & $60 / 50$ & $40 / 30$ & ns \\
\hline RDW (\%) & $14.5 \pm 2.1$ & $17.3 \pm 2.3$ & 0.011 \\
\hline $\begin{array}{l}\text { Etiology } \\
\text { biliary } \\
\text { alcohol } \\
\text { drugs } \\
\text { idiopathic }\end{array}$ & $\begin{array}{c}116(80.5 \%) \\
15(10.4 \%) \\
3(2.1 \%) \\
10(7 \%)\end{array}$ & $\begin{array}{l}27(75 \%) \\
4(11.1 \%) \\
1(2.8 \%) \\
4(11.1 \%)\end{array}$ & ns \\
\hline
\end{tabular}

AEP - acute interstitial edematous pancreatitis; ANP - acute necrotizing pancreatitis; RDW - red cell distribution width; ns - non-significant. 


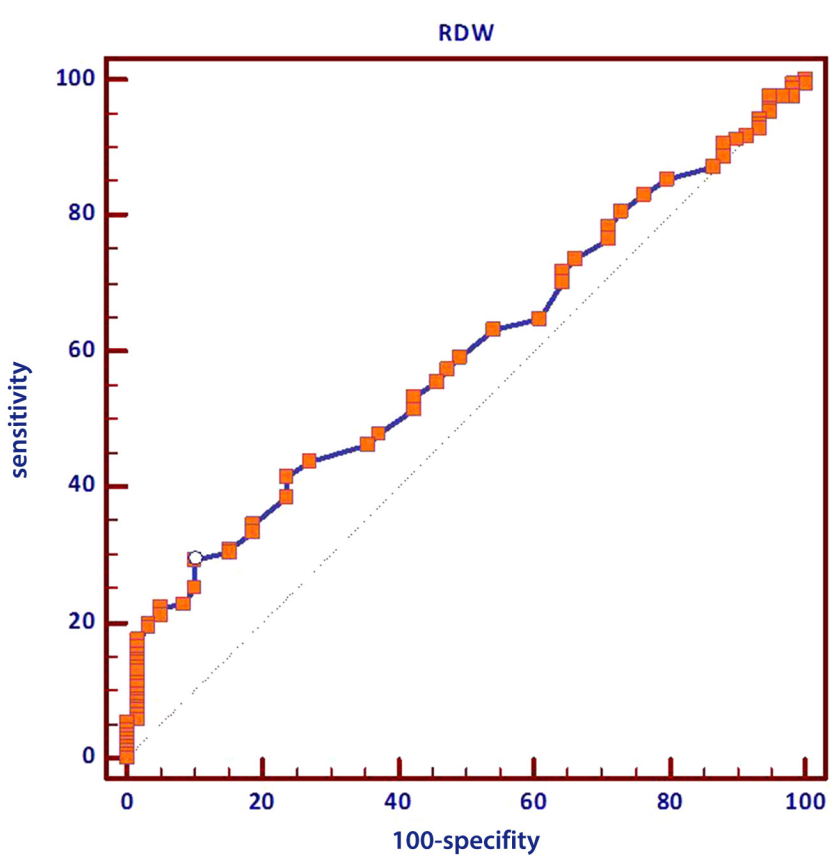

Fig. 1. The receiver operating characteristic (ROC) curve of red cell distribution width (RDW) values for predicting acute necrotizing pancreatitis in patients with acute pancreatitis

was no statistically significant difference between age, sex and etiologic factors in the patient groups. (Table 1).

Receiver operating characteristic (ROC) curve analysis was used to evaluate the values of RDW to diagnose necrotizing AP. The area under curve (AUC) and cut-off values were calculated. The AUC for the RDW value was 0.591 $(\mathrm{p}=0.0227)$. The cut-off value to diagnose necrotizing AP patients was 16.4 (sensitivity: 29.2\%, 95\% CI 22.5-36.7; specificity: 89.83\%, 95\% CI 79.2-96.2) (Fig. 1).

\section{Discussion}

There has been research to evaluate the relationship between RDW and mortality in patients with AP before. In our study, we found a relationship between the disease severity and the RDW values in patients with AP. Earlier studies included a small number of patients, while our study had the largest patient group.

RDW is calculated as part of complete blood count. It is used to distinguish the etiology of anemia and it shows heterogeneity. Normal values for RDW are 11.5-14.5\%. There is no case of RDW levels below normal limits. Thus, RDW is expressed as normal or high. Increased RDW shows a higher variation of erythrocyte size than normal. RDW is increased in hematologic and inflammatory diseases. The RDW value shows no sensitivity and specificity for a specific disease, and also does not exclude a diagnosis. RDW values are increased in hemolytic anemia, in the case of erythrocyte transfusion, and deficiency of vitamin $B_{12}$, folic acid and iron. . $^{911,12}$

Many studies have shown a correlation between higher
RDW values and prognosis, but these studies were about cardiovascular and cerebrovascular diseases, chronic renal failure, liver disease, pancreatitis and venous thromboembolism. ${ }^{13-17}$ Kurt et al. showed a correlation of nonvalvular atrial fibrillation and higher RDW values in 320 patients. ${ }^{13}$ Demir et al. conducted a study of 37 patients with cerebral sinus thrombosis and 101 patients with primary headache with a total of 138 patients. In that study, increased RDW values were correlated with the existence of vertebral sinus thrombosis. They showed that increased RDW values might be a predictor of cerebral sinus thrombosis in patients with primary headache. ${ }^{14}$ In a study of 367 patients with grade I-V chronic renal failure, Solak et al. reported a relationship between increased RDW values and flow mediated dilatation, which shows endothelial dysfunction independent of anemia, diabetes mellitus and inflammation. ${ }^{15}$ Kim et al. showed the correlation between increased RDW values and advanced fibrosis in 24,547 patients with nonalcoholic liver disease. ${ }^{16}$

The inflammation status of the disease may change the RDW values. The association of RDW with mortality may be explained by inflammation. Inflammation promotes the death of RBCs or inhibits the maturation of RBCs, thus decreasing RBC lifespan. RDW values may reflect the inflammation status of acute pancreatitis and may be used to predict severe acute pancreatitis. Bone marrow function and iron metabolism may be influenced by inflammation, and inflammatory mediators suppress erythrocyte maturation and cause larger and younger reticulocytes to enter the circulation, thus increasing RDW. Inflammation also increases oxidative stress leading to elevated RDW by reducing RBC survival and increasing the release of large, premature RBCs into the circulation. Inflammation itself also alters RBC membrane structure, contributing to changes in RBC morphology. ${ }^{17}$

Acute pancreatitis may cause local inflammation and also systemic effects. Mortality is higher in ANP than in AEP, so early diagnosis and treatment of ANP may improve the prognosis. ${ }^{17}$ At present, there is no single marker showing ANP. Biochemical tests, imaging studies and scoring systems are used in all clinics to detect the severity of AP, but 20-30\% of severe AP may not be diagnosed with these tests. ${ }^{18}$ An ideal marker - objective, simple, cheap, reproducible, sensitive and specific to AP, should be available in all clinics. ${ }^{17} \mathrm{RDW}$ is used to show the severity and prognosis of various diseases, and has been found to have the above-mentioned qualities. ${ }^{13-17}$ Wang et al. showed higher mortality rates with RDW values $>13.4 \% .{ }^{17}$ In our study, we found sensitivity of $88.2 \%$ and specificity of $91.8 \%$, with a cut-off value of $14.35 \%$ in ROC analysis. In a study including 103 patients, Şenol et al. observed that high RDW values on admission were associated with mortality. ${ }^{19}$ They found a cut-off value of $14.8 \%$ and predicted mortality in $77 \%$ of cases. They reported a sensitivity of $47.6 \%$ and a specificity of $96.3 \% .{ }^{19}$ Yao and Lv also reported a cut-off value of $14.2 \%$, and found higher RDW values in terminal 
patients with a sensitivity of $75 \%$ and specificity of $89.8 \%$ in 106 patients. ${ }^{20}$ Our study included 180 patients with AP. 144 patients had AEP, and 36 patients had ANP. We found that high RDW values on admission to the hospital were associated with severe disease. We found 16.4 as the cut-off value of RDW. Sensitivity was $29.2 \%$ and specificity was $89.83 \%$ for this value.

In conclusion, RDW can effectively distinguish ANP from AEP, and be used to evaluate the prognosis of AP. It is simple, cheap and reproducible.

\section{References}

1. Otsuki M, Takeda K, Matsuno S, et al. Criteria for the diagnosis and severity stratification of acute pancreatitis. World J Gastroenterol. 2013;19(35):5798-5805.

2. Stevenson K, Carter CR. Acute pancreatitis. Surgery (Oxford). 2013; 31(6):295-303.

3. Banks PA, Gerzof SG, Langevin RE, et al. Classification of acute pancreatitis - 2012: Revision of the Atlanta classification and definitions by international consensus. Gut. 2013;62(1):102-111.

4. van Santvoort HC, Bakker OJ, Bollen TL, et al. A Conservative and minimally invasive approach to necrotizing pancreatitis improves outcome. Gastroenterology. 2011;141(4):1254-1263.

5. Singh VK, Bollen TL, Wu BU, et al. An assessment of the severity of interstitial pancreatitis. Clin Gastroenterol Hepatol.2011;9(12):1098-1103.

6. Rau BM. Predicting severity of acute pancreatitis. Curr Gastroenterol Rep. 2007;9(2):107-115.

7. Zuidema MJ, van Santvoort HC, Besselink MG, et al. The predictive value of proteinuria in acute pancreatitis. Pancreatology. 2014;14(6): 484-489.

8. England JM, Down MC. Red-cell-volume distribution curves and the measurement of anisocytosis. Lancet. 1974;1(7860):701-703.
9. Fornal M, Wizner B, Cwynar M, et al. Association of red blood cell distribution width, inflammation markers and morphological as well as rheological erythrocyte parameters with target organ damage in hypertension. Clin Hemorheol Microcirc. 2014;56(4):325-335.

10. Hu ZD, Sun Y, Guo J, et al. Red blood cell distribution width is a potential index to assess the disease activity of systemic lupus erythematosus. Clin Chim Acta. 2013;425:202-205.

11. Aslan D, Gumruk F, Gurgey A, et al. Importance of RDW value in differential diagnosis of hypochrome anemias. Am J Hematol. 2002;69(1): 31-33.

12. AlFadhli SM, Al-Awadhi AM, AlKhaldi D. Validity assessment of nine discriminant functions used for the differentiation between iron deficiency anemia and thalassemia minor.J Trop Pediatr. 2007;53(2):93-97.

13. Kurt M, Tanboga IH, Buyukkaya E, et al. Relation of red cell distribution width with CHA2DS2-VASc score in patients with nonvalvular atrial fibrillation. Clin App/ Thromb Hemost. 2014;20(7):687-692.

14. Demir R, Saritemur M, Ozel L, Ozdemir G, Emet M, Ulvi H. Red cell distribution width identifies cerebral venous sinus thrombosis in patients with headache. Clin Appl Thromb Hemost. 2015;21(4): 354-358.

15. Solak Y, Yilmaz MI, Saglam M, et al. Red cell distribution width is independently related to endothelial dysfunction in patients with chronic kidney disease. Am J Med Sci. 2014;347(2):118-124.

16. Kim HM, Kim BS, Cho YK, et al. Elevated red cell distribution width is associated with advanced fibrosis in NAFLD. Clin Mol Hepatol. 2013; 19(3):258-265.

17. Wang $D$, Wang $R$, Liu $M$, et al. Red cell distribution width predicts deaths in patients with acute pancreatitis. J Res Med Sci. 2015;20(5): 424-428.

18. Guo ZH, Hao JY. The review of acute pancreatitis scoring system. Chin J Clin Hepatol. 2011;27:1170-1173 [in Chinese].

19. Şenol K, Saylam B, Kocaay F, et al. Red cell distribution width as a predictor of mortality in acute pancreatitis. Am J Emerg Med. 2013;31(4): 687-689.

20. Yao J, Lv G. Association between red cell distribution width and acute pancreatitis: A cross-sectional study. BMJ Open. 2014;4:e004721. 